\title{
Evaluation of Spectrum Occupancy in Amsterdam Using Mobile Monitoring Vehicles
}

\author{
Roel Schiphorst and Cornelis H. Slump
}

\begin{abstract}
Dynamic Spectrum Access (DSA) is an exciting new technology, which introduced a paradigm shift in spectrum access. As a result it also changes the role of the regulator. On one hand the scarce radio spectrum should be used in an optimal way, so that the society is best served. On the other hand interference between users and networks should be avoided. For that reason rules have to be defined for spectrum use, which are not only determined by technical parameters, also economical, legal, political and social constraints play an important role. This topic is called spectrum governance. For evaluation purposes a monitoring network is necessary, which is also supported by several papers, that argue the need for a monitoring network to assure compliance of (DSA) radio equipment with regulatory rules. Traditional networks of fixed monitoring sites are not qualified anymore, as modern communication use high frequencies, above the $800 \mathrm{MHz}$, and communicate mainly locally. In this paper, we propose to extent this monitoring network by a fleet of mobile monitoring vehicles.
\end{abstract}

Index Terms-Spectrum governance, dynamic spectrum access, monitoring network, spectrum measurements

\section{INTRODUCTION}

$\mathbf{T}$ $\mathrm{HE}$ radio spectrum is a relatively scarce resource and only frequencies between $200 \mathrm{MHz}$ and $3 \mathrm{GHz}$ are best qualified for wireless mobile communication. For that reason, rules have to be defined for spectrum use, which should have two goals; a) minimize interference between users and b) optimize the spectrum use. Of course, both goals are conflicting to some extent. A more efficient use of the radio spectrum can lead to more interference. These rules are not only determined by technical parameters, also economical, legal, political and social constraints play an important role in these rules. The topic of this research is called spectrum governance [1].

Traditional spectrum governance is focused on minimizing interference and locate (illegal) interferers, such as illegal FM radio transmissions. Radio propagation is another topic of interest. To fulfill these tasks, every nation has set up a network of monitoring sites to monitor the spectrum. However, the paradigm shift in spectrum access and its impact caused by DSA also require to change the traditional spectrum governance approach. As a result the traditional network of monitoring sites is not sufficient anymore and a new topology is necessary. In an ideal case, one would like to know the usage of the spectrum at every place, time and frequency. In principle one can compare it with a map for traffic density. In this case, the map should show the actual, real-time frequency usage in every area of a country.

The authors are with the University of Twente, Enschede, the Netherlands (e-mail \{r.schiphorst, c.h.slump\}@ewi.utwente.nl).

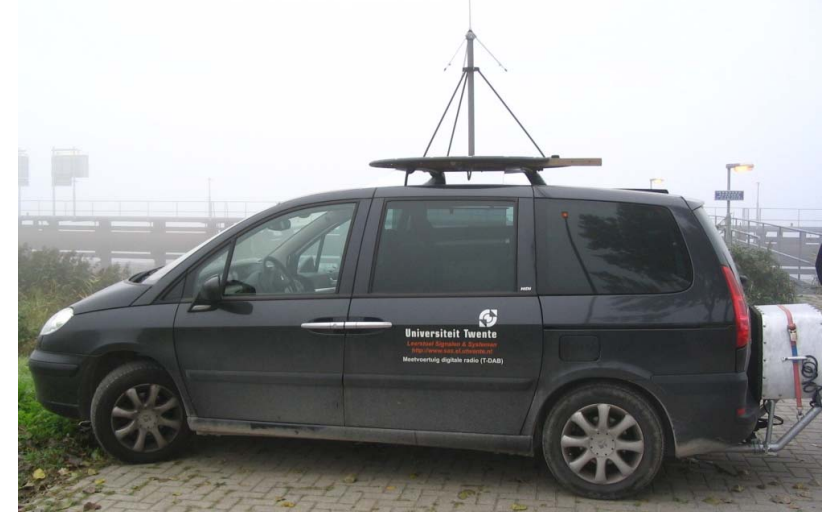

Fig. 1. Measurement vehicle.

This new monitoring network has several goals. First, it evaluates the performance of the current set of spectrum access rules, by measuring the spectrum use/occupancy and interference levels. Moreover, it can be used to check whether existing radio equipment complies with the rules. Also, the vast amount of measurement data can be used as input for DSA enabled radio to facilitate the search for the appropriate white space. Finally, it gives feedback to the current set of spectrum governance rules. Also it can be used to assure the compliance of DSA radio equipment with the regulatory rules [2], [3]. However, not much literature can be found how to design such a monitoring network. The design of this network is the topic of this paper.

The current monitoring network of fixed sites, is not sufficient for the new tasks of spectrum governance, because:

- Modern communication uses higher frequencies, up to 6 GHz.

- Several applications use two-way communication (base station - handset) e.g. GSM, UMTS, WiMAX. The uplink frequency is difficult to measure by the monitoring site network.

- The base stations of two-way communication use sectors and/or transmit beam forming. Directional transmission are also difficult to detect.

- Modern communication uses less transmit power and has a smaller service area per base station.

- Several applications use local communication without an infrastructure like wireless LAN (IEEE $802.11 \mathrm{a} / \mathrm{b} / \mathrm{g}$ ), Bluetooth. The spectrum use of these applications can not be measured with the current network.

In this paper, we present a new monitor network architecture 
that is tailored for the new tasks of spectrum monitoring. It consists of both fixed and mobile monitoring vehicles. Moreover, we describe and discuss first results based on the measurement campaign in Amsterdam that the University of Twente has carried out with the measurement vehicle shown in Figure 1. In addition, we describe new measurement methods to analyze the data that is collected by the network. Of course, this data can also be used by DSA-enabled radio to facilitate the search for white space. However, it will primary be an (important) tool for the regulator to monitor the efficiency of the current DSA policies and to adapt these, if necessary.

\section{MONITORING NETWORK}

In an ideal case, one would like to know the usage of the spectrum at every place, time and frequency. In principle one can compare it with a map for traffic density. Of course this is economically infeasible, because it would mean a dense network of fixed monitoring sites. Thus, it implies that the new monitoring network should measure especially where there are many people. The reason for this is that people use spectrum and therefore the highest spectrum needs are at places where there are many people.

Each application type requires a different approach of monitoring. For 'mission critical' applications like military, public safety and broadcasting services, interferers needs to be detected as soon as possible. For these kind of applications, a network of monitoring sites is required that measure real-time these bands " $24 / 7$ ". These services use frequency bands below $1 \mathrm{GHz}$ and are mostly infrastructure-based. So, the current 'traditional' network in the Netherlands is suitable to perform these tasks.

As we have stated in this paper, for other services like mobile communication, sensor networks and mobile internet such a network is not sufficient. Therefore, we propose in this paper to use mobile monitoring vehicles for these applications: a spectrum monitor mounted on a vehicle. For instance, they can be mounted on taxis or buses to get a good coverage in urban areas. In [4] a similar setup is described for the British radio communication agency, OFCOM, which focuses on the technical implementation. However, how to analyze this vast amount of data remains an open issue.

Benefits of mobile monitoring vehicles are:

- More mobile monitoring vehicles result in a better resolution.

- More mobile monitoring vehicles can be assigned to interesting areas.

- A mobile monitoring vehicle is very close to the actual users. Therefore it experiences the same spectrum as the users. Fixed monitoring sites measure a spectrum at a typical height of $50 \mathrm{~m}$.

- The standard deviation of radio signals in place is much larger than the standard deviation in time. So, to get a good overview of the spectrum, one would like to have as many monitoring sites as possible.

Several papers exists about measuring the white space in the radio spectrum [5]-[11], but all of them measure the spectrum at a single point. In our opinion this gives not a good impression of the actual use, as the parameter place is also important. This supported by our findings in [12], where we measured both the standard deviation in time and in place for a T-DAB signal. It turned out that the standard deviation in place is 3 to 8 times larger than the standard deviation in time. For the same reason, the hidden node problem is one of the challenges in cognitive radio. It can be mitigated by measuring at many places. So, for measuring the spectrum occupancy it seems appropriate to use the same approach. This is also reported for DSA systems in several papers [13]-[18] and supports the use of mobile monitoring vehicles.

In future, these mobile monitoring vehicle could be extended by multiple antennas to detect the direction of the radio signal. Onmidirectional antennas and software beam forming are a flexible solution for this application, compared to the use of directional antennas. Such a beam forming antenna array is described in [19].

In [18] a distributed spectrum monitoring network has been described for cognitive radio applications, where a feedback channel is used to calculate real time a spatiotemporal model of the spectrum using Gaussian process regression. Here, there is no feedback channel and all data is processed offline. Moreover, the main goal of the network described here is to serve the regulator and give a "mark" about the state of the radio spectrum in each geographic area. This is achieved by monitoring vehicles that are continuously moving. Important aspects are therefore: data reduction, computational efficient processing of collected data, and representation of spectrum use.

\section{SPECTRUM OCCUPANCY}

One of the most important questions for the regulator is to assess the spectrum usage. In this section we describe how to use the collected data of the mobile monitoring fleet. To assess the spectrum use, we propose to put a grid on the geographic area under investigation. A typical size of a square would be 10 by $10 \mathrm{~km}$, but its size depends on the application. In dense urban areas, the grid size can be chosen smaller and on the other hand in rural areas this size is too small. In each square, the measurements of all mobile monitoring vehicles are collected and we propose to pick $N$ random measurement points. In addition, the minimum distance between each point should be at least $M$ meters. In this way we get independent power spectrum measurement points and we prevent biasing of measurements points, when for instance a mobile monitoring vehicle is stuck in a traffic jam.

In the next step, we use spatial statistics on the measured data to assess the spectrum usage. Here, we assume that spectrum usage can be modeled by a log-normal distribution. Finally, questions about spectrum usage, interference level, etc. can be answered using statistical inference. In the next section, examples are given to illustrate this.

\section{A. Measurement results}

In Figure 2, the measured radio spectrum between $100 \mathrm{MHz}$ and $500 \mathrm{MHz}$ is depicted, during one measurement day. It contains about 300 spectrum traces that are measured with our 


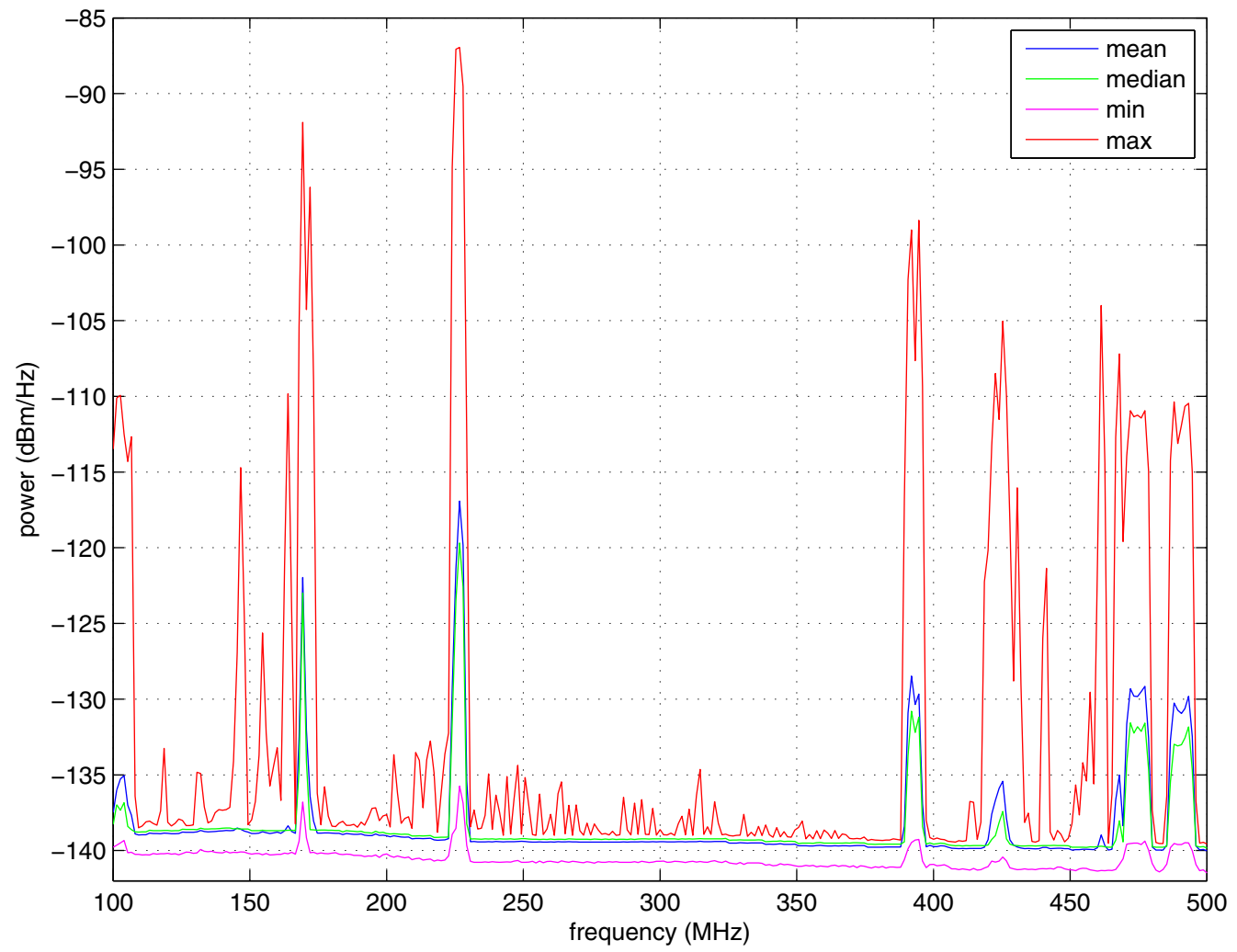

Fig. 2. The measured radio spectrum in Amsterdam.

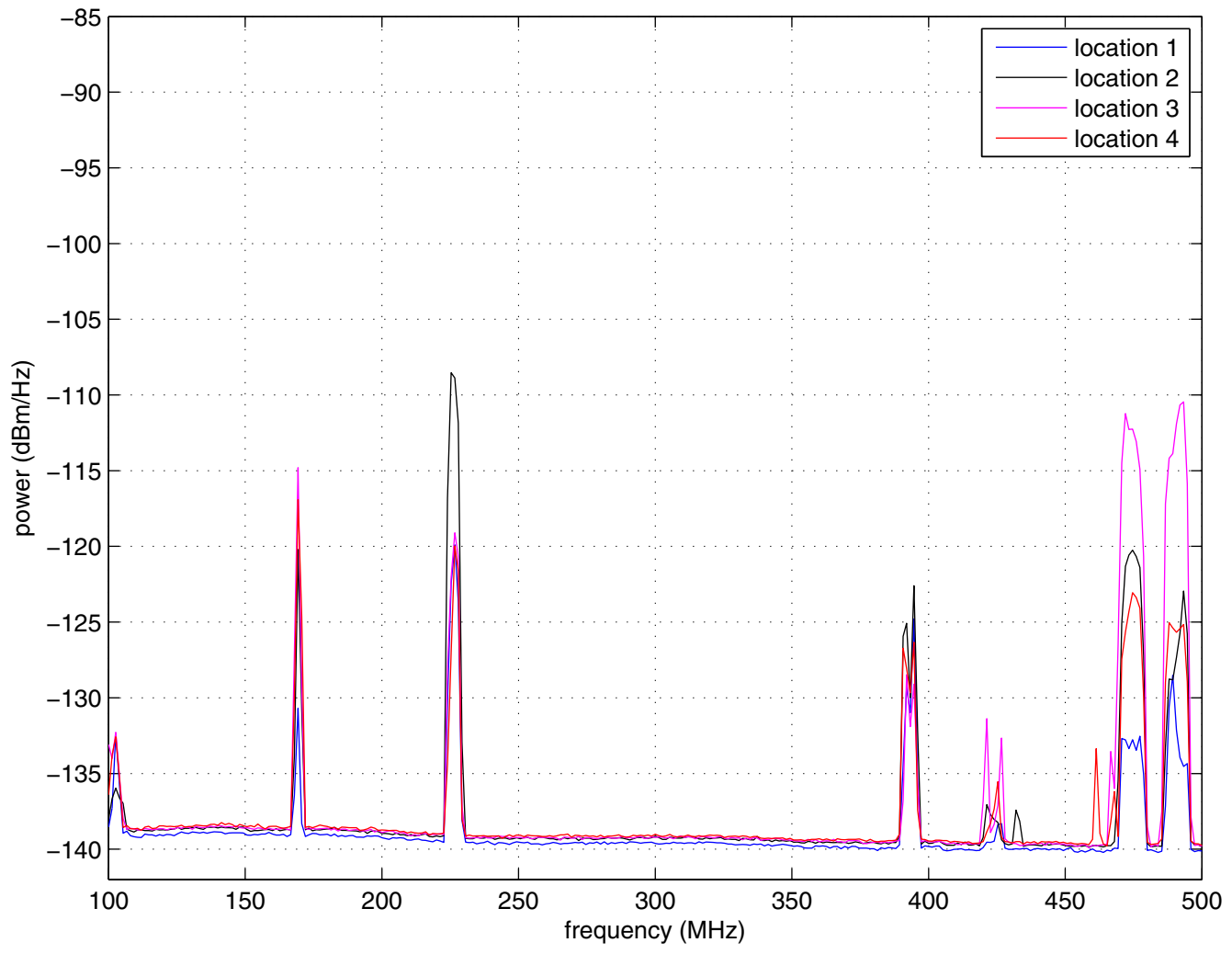

Fig. 3. The measured radio spectrum at several points in Amsterdam. 
measurement vehicle (Rhode \& Schwarz FSH), while driving through Amsterdam. More information about the measurement setup can be found in [12]. The FSH has a resolution bandwidth of $1.33 \mathrm{MHz}$ and all data is normalized to $\mathrm{dBm} / \mathrm{Hz}$.

Figure 2 shows the measured spectrum in Amsterdam and four lines are depicted: the mean, median, minimal and maximum value of each frequency bin. Several conclusions can be drawn from this figure. First, the noise floor of our measurement equipment is around $-140 \mathrm{dBm} / \mathrm{Hz}$. Second, the spectrum between $230 \mathrm{MHz}$ and $390 \mathrm{MHz}$ is used for military purposes. As expected there is almost no activity in this band. Outside this band, several frequency bands are in use. In these bands there is a huge difference up to $45 \mathrm{~dB}$ between the minimum and maximum values. This difference is much more than reported in papers, which measure the spectrum at a single point [5]-[11].

Thus by measuring at multiple points, the dynamic range of the received power spectra, is much larger compared to the received data of one point in time. Also, the figure reveals that for strong signals, there exists a difference of several dBs between the median and mean value. This indicates that the measured data contains extreme values and for that reason the median value should be used instead as mean value for the spectrum occupancy.

For reference purposes, Figure 3 depicts the measured power spectrum at 5 random locations. The data of these locations reveal that for a singe measurements, the spectrum is much more empty and also there is a large difference in the received signal strength for strong signals. (This is also shown in Figure 2.)

In the next step, we have put a grid on Amsterdam with a size of 3 by $3 \mathrm{~km}$ and used the measurement data of 5 days. Then, the collected data of both the public safety (around $400 \mathrm{MHz}$ ) and commercial trunked radio network (around $425 \mathrm{MHz}$ ) are fitted to a log-normal distribution and the accompanying CDFs are shown in Figure 4 and Figure 5 for five of these areas. (The numbers in the legend are the index to identify the area.)

In Figure 4, there is a small difference in the CDFs. This means that the spectrum occupancy and therefore use of the commerical trunked radio network is about the same in these areas. However, Figure 5 shows in an interesting result. The CDF of area_33 differs from the other areas and it reveals that in this area the public safety network is used less.

So, this method allows a quick and efficient overview of the spectrum use/occupancy in all geographic areas. Of course, it should be noted that the size of the geographic area can be dynamically assigned and more measurements and/or measurement vehicles will enhance the resolution of this data. So, overall this measurement setup is very flexible and can be tailored to the needs of the regulator.

In this example, the regulator may require from the commercial trunked radio network, that the $90 \%$ threshold is at least $-125 \mathrm{dBm} / \mathrm{Hz}$. If the measured value is lower, the license will be withdrawn, due to inefficient use. In addition, the regulator has identified from Figure 5 that the public safety network is used less in one area of Amsterdam. This may indicate that the network coverage in this area is insufficient.

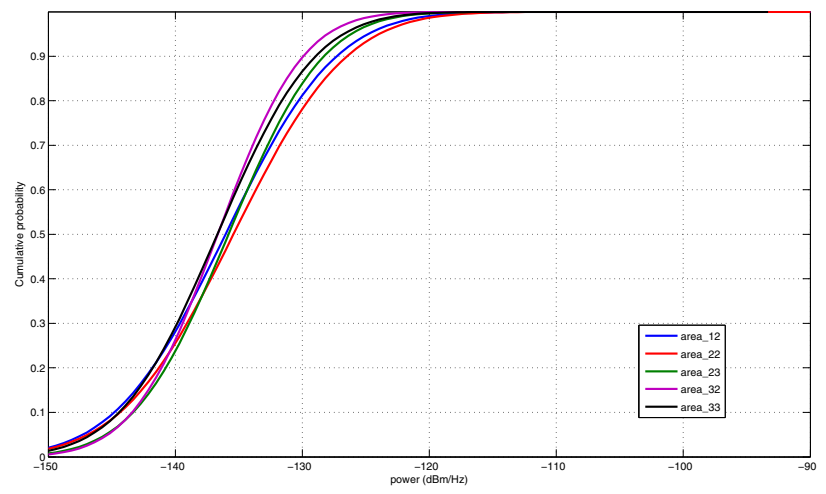

Fig. 4. CDFs of the commercial trunked radio network for different areas.

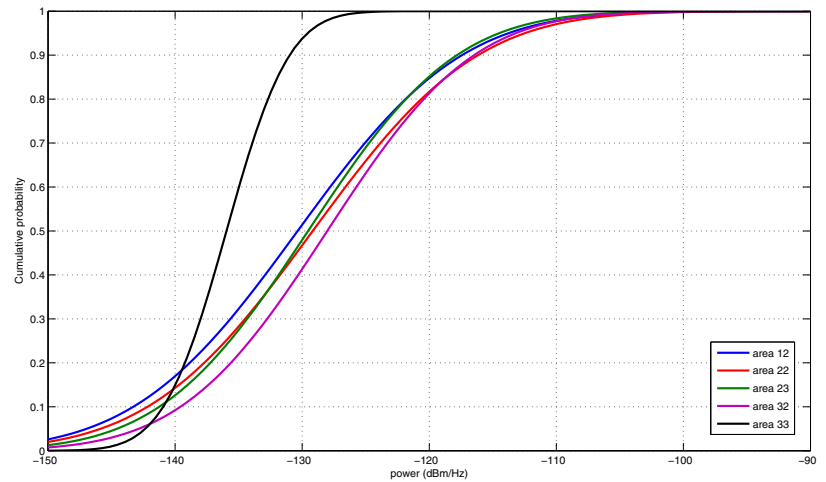

Fig. 5. CDFs of the public safety trunked radio network for different areas.

The primary goal of the monitoring network is to assist the regulator in fulfilling its tasks. However, we have stated in this paper that DSA and spectrum governance are not independent topics. The paradigm shift in spectrum access and its impact caused by DSA also require to change the traditional spectrum governance approach of "command and conquer". Feedback to the current spectrum governance rules are, among others, given by the output of the proposed monitoring network. On the other hand, the regulator collects with the proposed monitoring network, a vast amount of data about the spectrum usage. Of course, this data is not real-time and it only provides statistical information. So, DSA-enabled radio equipment can use this collected data, as a starting point for the search for unused spectrum.

\section{CONCLUSIONS AND FUTURE WORK}

In this paper we have described a new monitoring network for spectrum governance, which can also assist DSAenabled radios. It consists of fixed monitoring sites and mobile monitoring vehicles. The fixed monitoring sites are used for the 'traditional' tasks of spectrum governance such as locating interferers. However, for modern communication this monitoring network is not qualified anymore. The main reason for this is that modern communication use high frequencies, above the $800 \mathrm{MHz}$, and communicate mainly locally. Hence, a very dense network of monitoring sites would be required which is economically infeasible. Therefore, we have proposed to use mobile monitoring vehicles. In addition, we have described methods to analyze the measured data, which we 
have validated by measurements in the $100 \mathrm{MHz}$ to $500 \mathrm{MHz}$ band. The proposed method allows to determine the usage in a particular frequency band and geographic area. The size of this geographic are can be dynamically changed according to the needs of the regulator. Moreover, more measurements and measurement vehicles enhance the resolution of monitoring network.

In collaboration with the Dutch Radio communication agency, we use these initial results to initiate such a network of mobile monitoring vehicles. The system is based on the RFeye system of CRFS [20]. The RFeye system can be mounted on a normal vehicle and continuously measures the frequency spectrum to $6 \mathrm{GHz}$. All the data is stored on an storage USB key and the collected data is used for offline analysis. At the moment of writing, 10 RFeye systems are built into vehicles.

Further research aims at collecting more data, and analyze this data for a larger area. More research and data are also required to refine our proposed models and methods.

\section{ACKNOWLEDGMENTS}

The authors thank the Dutch radio communication agency for their support on this research.

\section{REFERENCES}

[1] W. Baumol and D. Robyn, Toward an Evolutionary Regime for Spectrum Governance: Licensing or Unrestricted Entry? Brookings Institution Press, 2006.

[2] A. Gad and F. Digham, "Impact of dsa on current regulatory regimes," in New Frontiers in Dynamic Spectrum Access Networks, 2008. DySPAN 2008. 3rd IEEE Symposium on, Oct. 2008, pp. 1-6.

[3] J. Bernthal, T. Brown, D. Hatfield, D. Sicker, P. Tenhula, and P. Weiser, "Trends and precedents favoring a regulatory embrace of smart radio technologies," in New Frontiers in Dynamic Spectrum Access Networks, 2007. DySPAN 2007. 2nd IEEE International Symposium on, April 2007, pp. 633-648.

[4] "Capture of spectrum utilisation information using moving vehicles," CRFS, Tech. Rep. Ofcom Tender No: 32/2008, 2009.

[5] M. Lopez-Benitez, A. Umbert, and F. Casadevall, "Evaluation of spectrum occupancy in Spain for cognitive radio applications," in Vehicular Technology Conference, 2009. VTC Spring 2009. IEEE 69th, April 2009, pp. 1-5.

[6] "http://www.sharedspectrum.com/measurements."
[7] M. Wellens, J. Wu, and P. Mahonen, "Evaluation of spectrum occupancy in indoor and outdoor scenario in the context of cognitive radio," in Cognitive Radio Oriented Wireless Networks and Communications, 2007. CrownCom 2007. 2nd International Conference on, Aug. 2007, pp. $420-427$.

[8] F. Sanders, "Broadband spectrum surveys in Denver, CO, San Diego, CA, and Los Angeles, CA: methodology, analysis, and comparative results," in Electromagnetic Compatibility, 1998. 1998 IEEE International Symposium on, vol. 2, Aug 1998, pp. 988-993 vol.2.

[9] S. Ellingson, "Spectral occupancy at vhf: implications for frequencyagile cognitive radios," in Vehicular Technology Conference, 2005. VTC2005-Fall. 2005 IEEE 62nd, vol. 2, Sept., 2005, pp. 1379-1382.

[10] M. Islam, C. Koh, S. Oh, X. Qing, Y. Lai, C. Wang, Y.-C. Liang, B. Toh, F. Chin, G. Tan, and W. Toh, "Spectrum survey in Singapore: Occupancy measurements and analyses," in Cognitive Radio Oriented Wireless Networks and Communications, 2008. CrownCom 2008. 3rd International Conference on, May 2008, pp. 1-7.

[11] R. Chiang, G. Rowe, and K. Sowerby, "A quantitative analysis of spectral occupancy measurements for cognitive radio," in Vehicular Technology Conference, 2007. VTC2007-Spring. IEEE 65th, April 2007, pp. 30163020.

[12] R. Schiphorst, N. Moseley, A. Aarden, M. Heskamp, and C. Slump, "A T-DAB field trial using a low-mast infrastructure," Broadcasting, IEEE Transactions on, vol. 54, no. 3, pp. 356-370, Sept. 2008.

[13] M. Wellens, J. Riihijrvia, and P. Mhnena, "Spatial statistics and models of spectrum use," Elsevier Computer Communications, Aug. 2009.

[14] D. Cabric, A. Tkachenko, and R. Brodersen, "Spectrum sensing measurements of pilot, energy, and collaborative detection," in Military Communications Conference, 2006. MILCOM 2006. IEEE, Oct. 2006, pp. 1-7.

[15] D. Cabric, A. Tkachenko, and R. W. Brodersen, "Experimental study of spectrum sensing based on energy detection and network cooperation," in TAPAS '06: Proceedings of the first international workshop on Technology and policy for accessing spectrum. New York, NY, USA: ACM, 2006, p. 12

[16] M. Wellens, J. Riihijarvi, M. Gordziel, and P. Mahonen, "Evaluation of cooperative spectrum sensing based on large scale measurements," in New Frontiers in Dynamic Spectrum Access Networks, 2008. DySPAN 2008. 3rd IEEE Symposium on, Oct. 2008, pp. 1-12.

[17] A. Ghasemi and E. Sousa, "Asymptotic performance of collaborative spectrum sensing under correlated log-normal shadowing," Communications Letters, IEEE, vol. 11, no. 1, Jan. 2007.

[18] J. P. Elsner, C. Koerner, and F. K. Jondral, "Centralized modeling of the communication space for spectral awareness in cognitive radio networks," SIGMOBILE Mob. Comput. Commun. Rev., vol. 13, no. 2, pp. 62-73, 2009.

[19] M. Kwakkernaat, Y. de Jong, R. Bultitude, and M. Herben, "Highresolution angle-of-arrival measurements on physically-nonstationary mobile radio channels," Antennas and Propagation, IEEE Transactions on, vol. 56, no. 8, pp. 2720-2729, Aug. 2008.

[20] "http://www.crfs.co.uk/." 